OPEN ACCESS

Edited by:

Attila Mócsai,

Semme/weis University, Hungary

Reviewed by:

Miguel Quiros,

University of Michigan, United States Áron Pánczél,

Semme/weis University, Hungary

*Correspondence:

Sandra Helena Poliselli Farsky sfarsky@usp.br

Specialty section:

This article was submitted to

Autoimmune and

Autoinflammatory Disorders,

a section of the journal

Frontiers in Immunology

Received: 24 May 2021

Accepted: 16 August 2021

Published: 17 September 2021

Citation:

de Paula-Silva M, da Rocha GHO, Broering MF, Queiroz ML, Sandri S, Loiola RA, Oliani SM, Vieira A

Perretti M and Farsky SHP (2021)

Formyl Peptide Receptors and

Annexin A1: Complementary

Mechanisms to Infliximab in

Murine Experimental Colitis

and Crohn's Disease.

Front. Immunol. 12:714138.

doi: 10.3389/fimmu.2021.714138

\section{Formyl Peptide Receptors and Annexin A1: Complementary Mechanisms to Infliximab in Murine Experimental Colitis and Crohn's Disease}

\author{
Marina de Paula-Silva ${ }^{1,2}$, Gustavo Henrique Oliveira da Rocha ${ }^{1}$, Milena Fronza Broering ${ }^{1}$, \\ Maria Luíza Queiroz ${ }^{3}$, Silvana Sandri ${ }^{1}$, Rodrigo Azevedo Loiola ${ }^{1}$, Sonia Maria Oliani ${ }^{4}$, \\ Andrea Vieira ${ }^{3}$, Mauro Perretti ${ }^{2}$ and Sandra Helena Poliselli Farsky ${ }^{1 *}$ \\ ${ }^{1}$ Department of Clinical and Toxicological Analyses, University of São Paulo (USP), São Paulo, Brazil, ${ }^{2}$ Centre for \\ Biochemical Pharmacology, The William Harvey Research Institute, Barts and The London School of Medicine, Queen Mary \\ University of London (QMUL), London, United Kingdom, ${ }^{3}$ Gastroenterology Service, Irmandade da Santa Casa de \\ Misericórdia de São Paulo, São Paulo, Brazil, ${ }^{4}$ Department of Biology, São Paulo State University (UNESP), São José do Rio \\ Preto, São Paulo, Brazil
}

Non-responsiveness to anti-TNF- $\alpha$ therapies presents relevant rates in inflammatory bowel disease patients, presenting the need to find biomarkers involved in therapeutic efficacy. Herein, we demonstrate that higher levels of colonic formyl peptide receptor 1 and annexin A1 correlate with histological recovery in Crohn's disease patients under remission. Using the dextran sulfate sodium colitis model in mice, we suggest that infliximab induces annexin A1 expression and secretion in activated intestinal leukocytes. Conversely, this mechanism might stimulate epithelial formyl peptide receptors, inducing wound healing and consequent histological remission. Our data indicate that assessing intestinal expressions of formyl peptide receptors and annexin A1 might provide precious information on the disease activity and responsiveness to infliximab in inflammatory bowel disease patients.

Keywords: biomarkers, formyl peptide receptor, annexin A1, infliximab, Crohn's disease, dextran sodium sulfate

\section{INTRODUCTION}

Inflammatory bowel diseases (IBDs), mainly Crohn's disease (CD) and ulcerative colitis (UC), are characterized by severe gastrointestinal inflammation (1). Biological therapies, such as monoclonal antibodies and infliximab (IFX), are very effective in inducing remission for moderate-to-severe IBDs $(2,3)$. By binding soluble and transmembrane tumor necrosis factor alpha (TNF- $\alpha$ ), IFX attenuates inflammation and decreases the need for surgery $(2,4,5)$. However, side effects and nonresponsiveness illuminate the relevance of validating biomarkers to assess therapeutic efficacy $(6,7)$.

We previously described a possible relationship between the response to IFX and the expression of annexin A1 (AnxA1) in mice with experimental colitis (8). AnxA1 is a resolutive mediator in human and experimental conditions such as cardiovascular diseases $(9,10)$, multiple sclerosis (11), 
and rheumatoid arthritis (12). AnxA1 is produced by epithelial cells and secreted by infiltrating leukocytes in IBD patients (13). Local expression of AnxA1 is pivotal to tissue recovery in $\mathrm{CD}$ and experimental colitis (14-16). Recently, it has been suggested that low levels of AnxA1 in CD support the uncontrolled inflammation that perpetuates the disease and that differential expressions of AnxA1 might allow the identification of disease severity patterns $(14,17)$. After cell activation, AnxA1 is mobilized to the membrane, where it is able to trigger antiinflammatory pathways through formyl peptide receptors (FPRs) (18). FPRs have been increasingly studied in IBD as they participate in antimicrobial and inflammatory processes. FPR expression in the gut correlates with pathology during acute inflammation, but plays a protective role in the chronic phases (19). FPR1 is a wound closure mediator $(20,21)$, whereas FPR2 induces mucosal healing by regulating the traffic of leukocytes into the inflamed tissue $(22,23)$.

This background provides valuable information about the roles of AnxA1 in IBD; however, only a couple of studies-including ours-have addressed the involvement of AnxA1 in the efficacy of IFX $(8,14)$. Also, none has explored the potential participation of the AnxA1-FPR axis. Herein, we describe some IFX mechanisms that are affected by the AnxA1-FPR axis and seek to explain how it could mediate distinct responses to anti-TNF- $\alpha$.

\section{MATERIALS AND METHODS}

\section{Patient Approach and Ethics Statement}

CD patients from the Santa Casa School of Medical Sciences (São Paulo, Brazil) and donors with no IBD history willingly donated blood (12 ml). Medical records and paraffinized colon biopsies provided the CD Activity Index (CDAI) (24) and microscopic grading (25). This study was conducted in accordance with the Declaration of Helsinki and the ethics boards from Santa Casa and the University of São Paulo (protocol \#07100819.3.0000.0067). Written consent was obtained.

\section{Criteria for Patient Enrollment}

The diagnosis of CD for patients participating in this study took into account endoscopic, histological, and clinical criteria assessed and interpreted by members of the medical staff from the Santa Casa School of Medical Sciences. Individuals younger than 18 years and/or with a diagnosis of an infectious disease (such as tuberculosis, chlamydia, and the common flu) were excluded.

Participants were divided into groups as follows:

Blood donors: Healthy individuals with no prior history of IBD provided control samples of blood matching the average age, gender, and ethnicity from CD groups. "Remission patients" reached clinical remission upon IFX treatment, while "Failure patients" were refractory to previous therapies and were not responding clinically to IFX at the moment of blood donation. One untreated CD patient provided active disease parameters and was receiving other medications (including immunosuppressants and corticosteroids), but not IFX.

Biopsy donors: Remission patients reached clinical and histological remission when treated with IFX. The Failure group was composed of patients who did not present improvement of clinical parameters and histological homeostasis upon IFX. "CD untreated" individuals were those with an active disease despite treatment administration of other medications (including immunosuppressants and corticosteroids), but not IFX.

It should be noted that all patients enrolled in this study had previously received or were receiving other medication classes at the time of our sample collection. The absence/interruption of responsiveness to other therapies followed by remission or nonremission upon IFX was the defining parameter for separating the Remission and Failure groups, respectively. Clinical parameters (mean and range of age, gender, and concomitant

TABLE 1 | Characteristics from $C D^{\mathrm{a}}$ patients and healthy donors.

\begin{tabular}{|c|c|c|c|c|c|c|c|}
\hline \multirow[t]{3}{*}{ Parameters } & \multirow{3}{*}{ Healthy donors } & \multicolumn{3}{|c|}{ Blood } & \multicolumn{3}{|c|}{ Tissue } \\
\hline & & \multicolumn{6}{|c|}{ CD patients } \\
\hline & & Untreated & Remission & Failure & Untreated & Remission & Failure \\
\hline Average age (mín.-max.) & $32(23-41)$ & 24 & $35(19-50)$ & 39 (19-63) & 28 (19-49) & $43(17-44)$ & $38(61-16)$ \\
\hline Standard deviation (age) & 10.11 & 0.00 & 12.14 & 14.65 & 13.96 & 3.51 & 31.81 \\
\hline Females & 3 & 1 & 8 & 5 & 3 & 2 & 0 \\
\hline Males & 2 & 0 & 4 & 7 & 1 & 1 & 2 \\
\hline Total & 5 & 1 & 12 & 11 & 4 & 3 & 2 \\
\hline \multicolumn{8}{|l|}{ Medications } \\
\hline Corticosteroids & - & - & - & $3(27.3 \%)$ & $1(25 \%)$ & - & 1 (50\%) \\
\hline Mesalazine & - & $1(100 \%)$ & $3(25 \%)$ & $6(54.6 \%)$ & $2(50 \%)$ & $2(66.6 \%)$ & 1 (50\%) \\
\hline Azathioprine & - & - & $3(25 \%)$ & $4(36.4 \%)$ & $3(75 \%)$ & - & $2(100 \%)$ \\
\hline Anti-diarrheic & - & - & $1(8.3 \%)$ & $1(9.1 \%)$ & - & $1(33.3 \%)$ & - \\
\hline Antidepressants & $2(40 \%)$ & - & $2(16.6 \%)$ & - & - & $1(33.3 \%)$ & $1(50 \%)$ \\
\hline Antibiotics & - & - & - & $2(18.2 \%)$ & $1(25 \%)$ & - & - \\
\hline Hepatics and pancreatics & - & - & - & $3(27.3 \%)$ & $1(25 \%)$ & - & $1(50 \%)$ \\
\hline Hepatics and Hidrocortisone pre-IFX ${ }^{b}$ & - & - & - & $1(9.1 \%)$ & - & - & - \\
\hline Other & 2 (40\%) & $1(100 \%)$ & 2 (16.6\%) & 4 (36.4\%) & 2 (50\%) & 1 (33.3\%) & 2 (100\%) \\
\hline
\end{tabular}

${ }^{a} \mathrm{CD}$, Crohn's disease; ${ }^{b} \mathrm{IFX}$, infliximab. 
medications) from the Untreated CD, CD+IFX Remission, or CD+IFX Failure groups are presented in Table $\mathbf{1}$.

\section{Isolation of Leukocytes and Detection of AnxA1 in the Blood of CD Patients}

Blood from healthy donors $(n=5)$ and CD patients treated with IFX ( $n=23)$ or not $(n=1)$ was used to isolate the following:

Plasma AnxA1: AnxA1 was detected in plasma samples using an ELISA kit (MyBioSource, San Diego, CA, USA).

Leukocytes: $\mathrm{NH}_{4} \mathrm{Cl}(0.13 \mathrm{M})$ was added to the remaining cell fraction to lysate erythrocytes. Pellets were fixed in $1 \%$ paraformaldehyde (PFA) and incubated with anti-FPR1 (PE, R\&D Systems, Minneapolis, MN, USA) and anti-FPR2 (FITC, Bioss, Woburn, MA, USA) antibodies. Readings were conducted using a BD Accuri Flow Cytometer (BD Biosciences, Franklin Lakes, NJ, USA) to acquire 10,000 events/sample. Positive populations were determined by labeling for each antibody separately.

\section{Detection of AnxA1, FPR1 and FPR2 in CD Biopsies}

Paraffin-embedded colon biopsies from CD untreated $(n=4)$ and treated positive $(n=3)$ or negative $(n=2)$ responders to IFX were permeabilized $(0.01 \%$ Triton), retrieved (sodium citrate buffer), and blocked (20\% fetal bovine serum, FBS). Samples were incubated with mouse anti-FPR1 (1:25, clone 5F1; BD Biosciences), anti-FPR2 (1:10, clone 2D8; Sigma-Aldrich, St. Louis, MO, USA), or anti-AnxA1 (1:50, clone $1 \mathrm{~B}, 10 \mu \mathrm{g} / \mathrm{ml})$. Incubation with $20 \%$ FBS provided the negative control. After incubation with anti-mouse Alexa Fluor 488 secondary antibody (1:200; Thermo Fisher, Waltham, MA, USA) and DAPI, the slides were mounted and five regions of interest (ROIs) per slide were photographed on a confocal microscope (Zeiss LSM800). Before acquiring images, the settings for gain, offset, and exposure time were adjusted based on the reaction control and standardized for each ROI from the stained samples. Acquired composite images (.czi format) were imported to Fiji (ImageJ Software, Bethesda, MD, USA) and split into blue and green channels. For densitometric analysis, the green channel (Alexa Fluor 488) was selected and modified to be displayed with a gray filter. Background pixel averages were then subtracted from the image pixels of interest to correct uneven illumination with the aid of the "Process $>$ Math $>$ Subtract" process. Fluorescence measures were performed manually by the selection of positive regions; average values were expressed in arbitrary units.

\section{Ethics Statement and Animals}

C57BL6 wild-type (WT) or AnxA1-null mice $\left(\mathrm{AnxA1}^{-/-}\right)$, males, 8-10 weeks old, were used to perform colitis. WT C57BL6, males, 16-18 weeks old, were used to provide intestinal immune cells to ex vivo experiments. Mice were obtained from the Federal University of São Paulo Animal House (Brazil), kept in 12:12-h light/dark cycle, and provided with food and water ad libitum. Experiments were performed in accordance with the Brazilian laws of protection and approved by the Committee on Ethics of Animal Experiments from the University of São Paulo.

\section{Colitis Model and Clinical Analysis}

Fresh 2\% dextran sodium sulfate (DSS; weight/volume, $40 \mathrm{kDa}$; Dextran Products Limited, Scarborough, Ontario, Canada) was added to the drinking water of WT and $A n x A 1^{-1-}$ mice (day 0 ) and replenished every other day up to day 6 (26). Control and non-treated DSS mice received vehicle (sterile saline $0.9 \%+$ DMSO 0.5\%) intraperitoneally (i.p.) on days 0-9. DSS-treated groups received i.p. IFX (1 mg/kg; Remicade ${ }^{\circledR}$ Janssen-Cilag, Buenos Aires, Argentina) on day 1 and/or FPR antagonist Boc-2 $(10 \mu \mathrm{g} / \mathrm{kg} ; \mathrm{N}-\mathrm{t}-\mathrm{BOC}-\mathrm{MET}-\mathrm{LEU}-\mathrm{PHE}, \mathrm{MP}$ Biomedicals, Irvine, CA, USA) on days 0-9. Body weight, diarrhea, and rectal bleeding were scored daily to provide the Disease Activity Index (DAI). On day 10, mice were euthanized by overexposure to nasal anaesthesia [isoflurane; 2-chloro-2(difluoromethoxy)-1,1,1-trifluoro-ethane].

\section{Dosage of Tissue MMP-9}

Samples of medium colon were homogenized in RIPA buffer containing a protease inhibitor (1:100; Thermo Fisher) in an Ultra-Turrax homogenizer (T10-Basic-IKA). After $20 \mathrm{~min}$ on ice, the tissue debris were removed and matrix metalloproteinase 9 (MMP-9) was detected by ELISA (R\&D Systems).

\section{Histological Analysis In Vivo}

Distal colons were fixed in $4 \%$ buffered PFA, dehydrated, and embedded in paraffin. Samples were analyzed using high-power objectives on the Imager.A2 Zeiss microscope (Zeiss, Oberkochen, Germany).

\section{Histopathology}

Histological grading was based on a previous report (8). The following features were analyzed by a blinded histologist: changes on crypts and histoarchitecture, edema, ulceration and immune cells at the epithelium, lamina propria, or submucosa. Grades of $0,1,2,3$, and 4 were respectively attributed to normal, mild, mild-moderate, moderate-severe, and severe conditions. Results were expressed as the mean of total grading.

\section{Immunohistochemistry}

After permeabilization (Triton 0.01\%) and antigen retrieval (sodium citrate buffer, $10 \mathrm{mM}, \mathrm{pH} 6.0$ ), peroxidase was inactivated with $3 \%$ hydrogen peroxide. Samples were blocked with $10 \%$ Tris-buffered saline-bovine serum albumin and incubated with the anti-AnxA1 antibody (1:500; Thermo Fisher). The reaction control was incubated with a blocking solution. The slides were finalized with an anti-rabbit horseradish peroxidase (HRP) antibody (Abcam, Cambridge, UK), 3,3-diaminobenzidine (DAB; Thermo Fisher), and hematoxylin counterstaining.

\section{Immunofluorescence}

Permeabilized samples were retrieved in sodium citrate buffer and blocked with 20\% FBS. Antibodies were incubated overnight $\left(4^{\circ} \mathrm{C}\right)$ : polyclonal mouse anti- $\beta$-actin $(1: 200)$ and rabbit antivillin (1:50; Abcam). After secondary antibodies (anti-mouse DyLight 549 and anti-rabbit FITC, 1:200; Vector Laboratories, Burlingame, CA, USA), the nuclei were stained with DAPI. 


\section{Isolation of Leukocytes From Lamina Propria and Flow Cytometry}

Leukocytes from proximal colonic lamina propria were isolated after washes with $2 \mathrm{mM}$ EDTA and digestion with collagenase $\mathrm{V}$ from Clostridium histolyticum (1 mg/ml; Sigma-Aldrich) (26). The cells were washed through $40-\mu \mathrm{m}$ strainers (Corning, Corning, NY, USA) and stained with CD4 (FITC) and CD25 (APC) (1:100; BD Biosciences). Positive populations were determined by labeling with single antibodies. A minimum of 10,000 events per sample were acquired on a BD Accuri Flow Cytometer. The results were expressed as percentages of positive cells normalized by controls from each experiment.

\section{Isolation of Colonic Lamina Propria Leukocytes and Ex Vivo Treatments}

After euthanasia by overexposure to isoflurane, colons from C57BL6 mice were opened longitudinally and washed with supplemented phosphate-buffered saline $(10,000 \mu \mathrm{g} / \mathrm{ml}$ penicillin/ streptomycin and $50 \mu \mathrm{g} / \mathrm{ml}$ gentamycin). Under a sterile hood, the tissues were fragmented, washed in Hank's salt solution buffer without calcium/magnesium for $20 \mathrm{~min}$ (twice), and digested with collagenases from C. histolyticum (types II and IV, $0.5 \mathrm{mg} / \mathrm{ml}$; Gibco, Waltham, MA, USA). The digested tissue was washed twice through $40-\mu \mathrm{m}$ strainers (Corning) and the pellets were counted and resuspended at the Roswell Park Memorial Institute (RPMI + $1 \%$ FBS). Cells were seeded in a 96-well plate $\left(2 \times 10^{5} /\right.$ well $)$ and treated with $200 \mathrm{ng} / \mathrm{ml}$ lipopolysaccharide (LPS; Sigma-Aldrich) 30 $\min$ before $\operatorname{IFX}(0.1,1.0$, or $10.0 \mu \mathrm{g} / \mathrm{ml})$. Controls were untreated or treated with those IFX doses. After $24 \mathrm{~h}$, the supernatants were collected to dose secreted AnxA1 (MyBioSource) according to the manufacturer's instructions.

\section{Statistical Analysis}

To determine the parametric or non-parametric distributions, we used the Kolmogorov-Smirnov test. ANOVA followed by Tukey's was performed for parametric tests, and KruskalWallis followed by Dunn's post-test was performed for nonparametric tests. To compare the two groups, we applied unpaired (single measures) or paired $t$-test (repeated measures). Pearson's correlation was performed for correlation analysis, providing the correlation coefficient $r$ and the coefficient of determination $R^{2}$. Probabilities with $p<0.05$ were considered significant. The results were expressed as the mean \pm standard deviation (SD; human samples, individual variabilities) or standard error of the mean (SEM; in vivo/ex vivo assays, group variability). All statistical assessments were conducted using GraphPad Prism ${ }^{\circledR}$ software, version 9.1.2 (San Diego, CA, USA).

\section{RESULTS}

\section{AnxA1 and FPR1 Are Differentially Expressed in the Colon of CD Patients Responsive to IFX}

Recently, it has been suggested that differential expressions of AnxA1 might allow the identification of disease severity patterns in IBD $(14,17)$. Thus, we proposed screening the expression patterns of AnxA1 and its receptors, FPR1 and FPR2, in CD biopsies of colons from untreated and IFX-treated patients who either went into remission or did not. Information about the healthy volunteers and CD patients are listed in Table $\mathbf{1 .}$

Hematoxylin/eosin-stained colon biopsies revealed ulcers, crypt alterations/abscesses, and prominent inflammatory infiltrates in untreated and IFX Failure patients (Supplementary Figures 1A, C). Based on the grading system (Supplementary Table 1), we confirmed lower histological damage for remittent (Supplementary Figure 1B and Figure 1F) rather than untreated $\mathrm{CD}$ individuals.

Subsequently, FPR1, FPR2, and AnxA1 were detected on the colon biopsies using confocal microscopy (Figure 1). For those three markers, the $Z$-scores were above the population mean for the IFX-induced remission group, contrasting with the lower levels in the untreated and IFX Failure groups (Figure 1A). Despite the increased fluorescence detected for FPR1 (Figures 1C, G) and FPR2 (Figures 1D, H) in remittent patients, only AnxA1 was significantly higher than that in the untreated group (Figures 1E, I). Epithelial cells from barrier, crypts, and leukocytes were the major sources of FPR1, FPR2, and AnxA1. Furthermore, colonic FPR1 and AnxA1 expressions presented a strong negative correlation with the histological grading (Figures 1J, L), which means that the decrease of these markers is associated with more tissue damage. For FPR2, a moderate correlation was detected $(r=-0.5355)$, but this was not enough to characterize a significant association (Figure 1K). Based on this, we presume that the upregulation of colonic FPR1 and AnxA1 in CD positive responders to IFX provides a marker of differential expression for therapy efficacy.

Using the patients' medical histories, we calculated the CDAI (Supplementary Table 2). Despite the different intensities of disease activity among patients, no patterns for circulating FPR1, FPR2 (leukocytes), or AnxA1 (plasma) were observed for the healthy, remission, or failure groups (Supplementary Figure 2A). As expected, CDAI was significantly higher in IFX-unresponsive patients than in remission patients (Supplementary Figure 2B). Furthermore, FPR1, FPR2, and AnxA1 in blood did not correlate with CDAI, indicating that patients' circulating levels might not be reliable biomarkers for remission or failure after IFX treatment (Supplementary Figures 2C-H).

\section{FPR Signaling Complements the Beneficial Effects of IFX on Colitis Symptomatology and Tissue Damage}

After detecting the correlations between high expressions of FPR1/AnxA1 and histological recovery in CD, we wondered how the modulation of these markers would impact resolution after IFX. We have previously demonstrated the failure of IFX in treating acute colitis using AnxA1-null Balb/c mice (8). To explore the mechanisms involved in the efficacy of IFX, we performed DSS-induced colitis in WT and AnxA1 ${ }^{-1-}$ C57BL6 mice and followed up to the late phase of the disease (Figure 2 and Supplementary Figures 3, 4). As expected, colitis induced significant weight loss and increased the DAI in both WT (Figures 2A-C) and AnxA1 ${ }^{-/-}$strains (Supplementary 

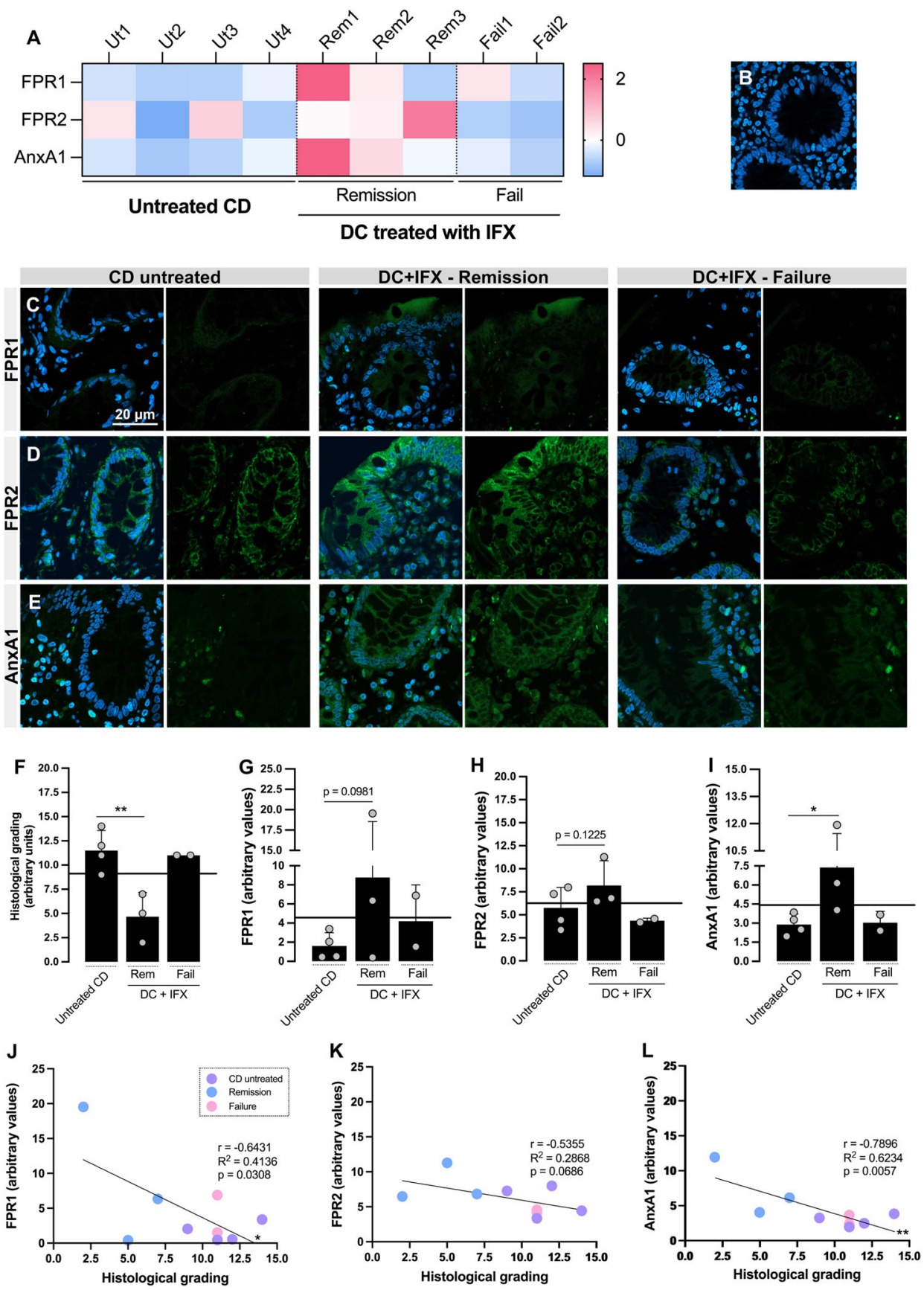

FIGURE 1 | Formyl peptide receptor 1 (FPR1), FPR2, and annexin A1 (AnXA1) are differentially expressed in the colon of Crohn's disease (CD) responders to infliximab (IFX) and correlate with histological homeostasis. (A) Heatmap based on the Z-scores of FPR1, FPR2, and AnxA1 expression values in the colon. (B-E) Confocal imaging: negative control of reaction (B), FPR1 (C), FPR2 (D), and AnXA1 (E) tissue staining. Embedding, paraffin; sections, $3 \mu \mathrm{m}$. Bar, $20 \mu \mathrm{m}$. (F) Histological grading, calculated based on Supplementary Table 1. (G-I) Densitometric analysis of fluorescence: FPR1 (G), FPR2 (H), and AnXA1 (I). (J-L) Correlation analysis between histological grading of biopsies and fluorescence intensity of staining: FPR1 (J), FPR2 (K), and AnXA1 (L). ${ }^{*} p<0.05,{ }^{* *} p<0.01 . n=4$ (CD untreated); $n=3$ (Remission); $n=2$ (Failure). Results are expressed as the mean \pm SD.

Figures 4A, B). Anatomic and microscopic alterations were also produced by DSS, including colon shortening, MMP-9 increase, ulcers, altered crypt, crypt abscesses, vacuolar hydropic degeneration, submucosal edema, and mucosal/submucosal massive inflammatory infiltrates (Figures 2D-H and Supplementary Figures 4E-I). IFX attenuated body weight loss (days 9 and 10; Figure 2A) and DAI during the late phase of the disease (day 8; Figures $\mathbf{2 B}, \mathbf{C}$ ) in WT mice. Except for 

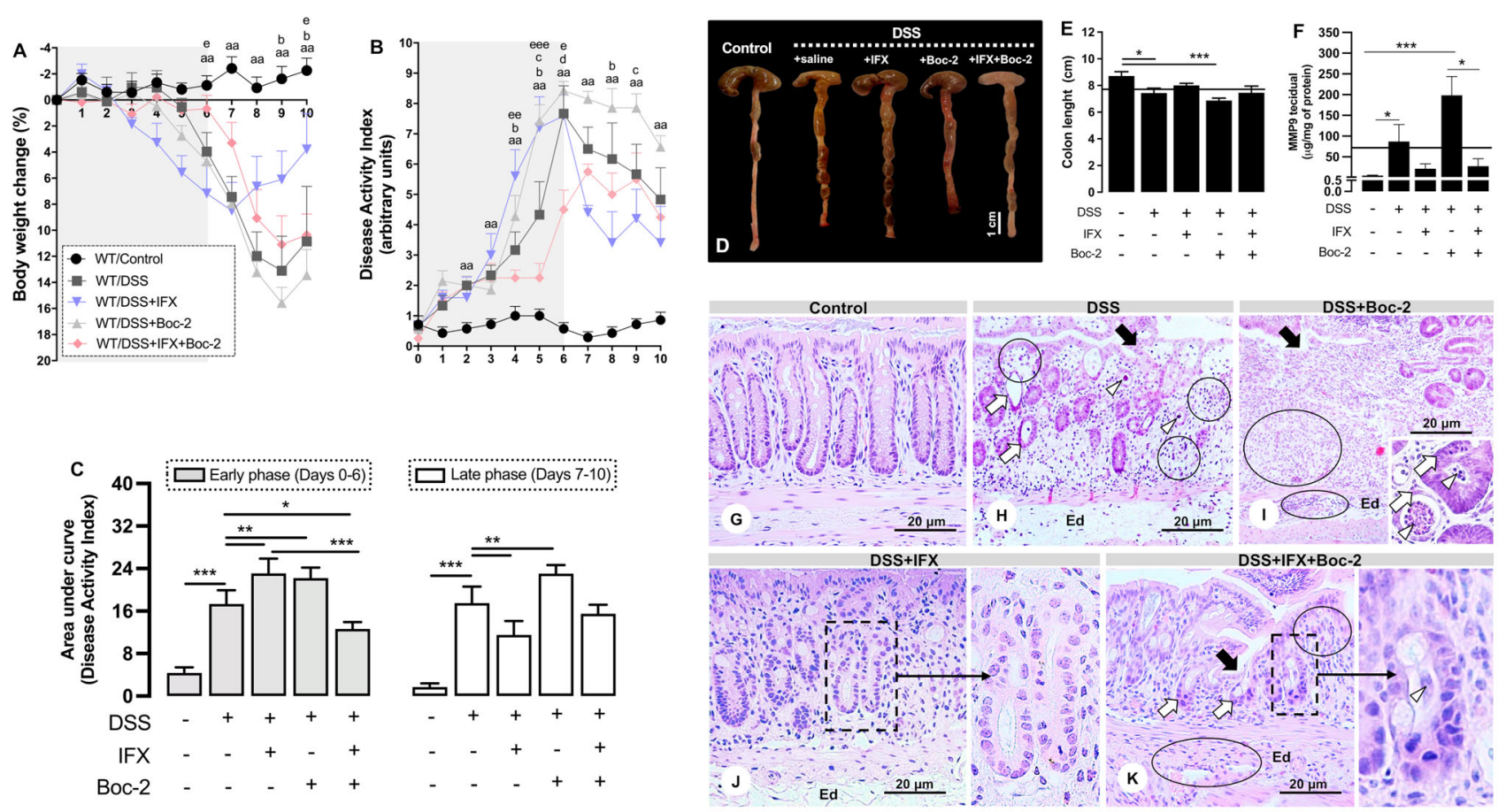

FIGURE 2 | Formyl peptide receptor (FPR) blockade impairs clinical and histological improvements induced by infliximab (IFX) during colitis. (A) Loss of body weight. (B) Disease Activity Index (DAl). (C) Area under the curve from DAl, early and late phases. (D, E) Colon length and anatomic changes. (F) Colonic MMP-9 levels. Mean of all samples (continuous lines). Bar, 1 cm. ${ }^{a a} p<0.01$ (DSS vs. Control); ${ }^{b} p<0.05$ (DSS+IFX vs. DSS); ${ }^{p} p<0.05$ (DSS+Boc-2 vs. DSS); ${ }^{d} p<0.05$ (DSS+IFX+Boc-2 vs. DSS); ${ }^{e} p<0.05,{ }^{e} p<0.01,{ }^{e e} p<0.001$ (DSS+IFX+Boc-2 vs. DSS+IFX). ${ }^{*} p<0.05,{ }^{\star \star} p<0.01,{ }^{\star \star \star} p<0.001 . n=4-6$ mice/group. Results are expressed as the mean \pm SEM. (G-K) Histopathology in wild-type (WT) groups. Ulcer (black arrows), altered crypts (white arrows), crypt abscesses (white arrowheads), edema (Ed), and inflammatory infiltrate (circles). Staining, hematoxylin-eosin; embedding, paraffin; sections, $3 \mu \mathrm{m}$. Bars, $20 \mu \mathrm{m}$.

some remaining infiltrated leukocytes and submucosal edema, the colonic histological architecture was protected by IFX (Figure 2J). These improvements were not only absent in the $\mathrm{AnxA1}^{-1-}$ group upon IFX treatment (Supplementary Figures 4A, B, J), but anti-TNF- $\alpha$ seemed to be harmful without endogenous AnxA1 as well: a 50\% mortality rate was recorded (Supplementary Figure 4C). AnxA1-deficient mice had significant colon shortening (Supplementary Figures 4E, F) and a 200- to 300-fold increase in tissue MMP-9 with or without IFX treatment (Supplementary Figure 4G). Moreover, AnxA1 $1^{-/-}$DSS+IFX mice had less intestinal $\mathrm{T}$ regulatory lymphocytes than did the mice in the respective WT group and the untreated group (AnxA1 $1^{-/} / \mathrm{DSS}$ ), reaffirming the relevance of AnxA1 to the downregulation of inflammation even when TNF- $\alpha$ is being blocked by IFX (Supplementary Figure 4D).

For the next step, we blocked the AnxA1 receptors, FPRs, to assess their potential involvement in the effects of IFX. The FPR blockade, by itself, confirmed their well-known role in wound healing $(15,27)$. FPR neutralization was detrimental to the clinical parameters in both early and late phases of disease (Figures 2A-C), intensified colon shortening (Figures 2D, E), and increased MMP-9 (Figure 2F). Microscopically, the FPR blockade with Boc-2 worsened the DSS damage, especially with regard to epithelial/glandular loss and infiltrated leukocytes
(Figure 2I). When combined with IFX, blocking FPRs was detrimental to the clinical conditions in the late phase as the beneficial effects of IFX on body weight and DAI were lost (days 9 and 10 and day 8 in Figures 2A, B, respectively) (Figure 2C). Although the colon length and MMP-9 from IFX-treated mice were not affected by Boc-2 (Figures 2D, E), the cecum very consistently assumed a distorted morphology and paleness for this group (Figure 2D).

Finally, the histological parameters were analyzed. IFXmediated preservation of the colonic histoarchitecture was partially impaired by an FPR blockade, with the presence of immune cells in the mucosa and punctual but persistent ulcerations (Figure 2K). The clearest histological alteration in this group was poor crypt recovery (details in Figure $\mathbf{2 K}$ ). For this reason, we proceeded to explore the effects of halting the signaling through FPRs for the regeneration of crypts after IFX treatment.

\section{Blocking FPRs Compromises the Effects of IFX on Crypt Regeneration}

The relevance of FPRs for crypt regeneration was confirmed by structural protein immunostaining (Figure 3). In control mice, $\beta$-actin revealed the cylindric shape of enterocytes (Figure 3A, a1) with villin expressed on the apical surfaces of cells (Figure 3A, a2). A similar pattern was observed on crypts 


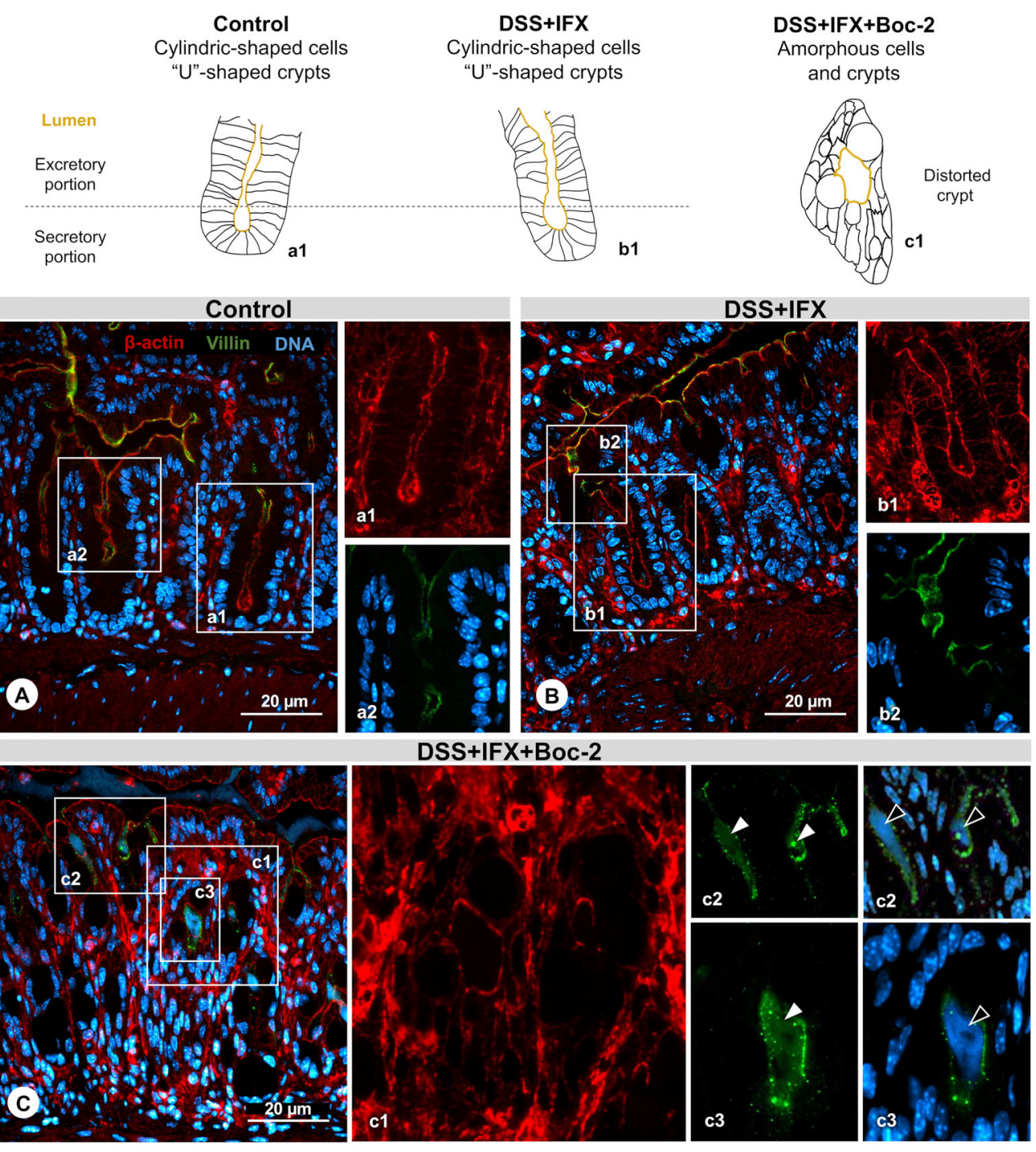

FIGURE 3 | Crypt regeneration mediated by infliximab (IFX) requires formyl peptide receptor (FPR) signaling. (A) Control: cylindrical-shaped cells forming a U-shaped crypt revealed by $\beta$-actin staining (a1, red). Presence of villin (a2, green) in the inner surface from the excretory part of the crypt lumen. Representation of a normal structure. (B) DSS+IFX: preserved crypt morphology resembling the control condition (b1 and b2). (C) DSS+IFX+Boc-2: defects in crypt closure (c1); presence of villin (c2 and c3, white arrowheads); and DNA residue (c2 and c3, black arrowheads) in the crypt lumen. $n=4-6$ mice/group. Embedding, paraffin; sections, $3 \mu \mathrm{m}$. Bars, $20 \mu \mathrm{m}$.

from DSS+IFX mice (Figure 3B, b1 and b2). In both groups, it is possible to identify the secretory and excretory portions from the mucosal glands, forming a U-shaped structure with a centered vertical lumen that opens to the intestinal wall, where the secretions produced by crypts are liberated.

Upon FPR inactivation, with or without IFX treatment, crypt cells assumed a deformed morphology lacking cylindric limits. The elongated shape from crypts gave place to an amorphous structure, many times with the lumen turned into a round form that was no longer connected with the epithelium surface (Figure 3C, c1). In this space, we observed residues of the shed villin (Figure 3C, c2 and c3, green) that co-localized with DNA (Figure 3C, c2 and c3, blue). FPRs were associated with epithelial repair, migration, and wound healing before, but our results suggest that these roles are also required for the intestinal barrier homeostasis mediated by IFX. Considering that AnxA1 is an important FPR agonist and a pivotal mediator of tissue repair in IBD, our final step was to assess the possible kinds of interplay among AnxA1 expression, FPR activation, and IFX efficacy.

\section{IFX Induces AnxA1 Expression and Secretion by Tissue Leukocytes During Inflammation}

Intracellular and FPR-binding AnxA1 mediate inflammation resolution and tissue repair on experimental colitis $(8,28)$. In our model, we assessed the expression patterns for endogenous AnxA1 in the colon (Figure 4). The reaction control confirmed specificity (Figure 4J). 

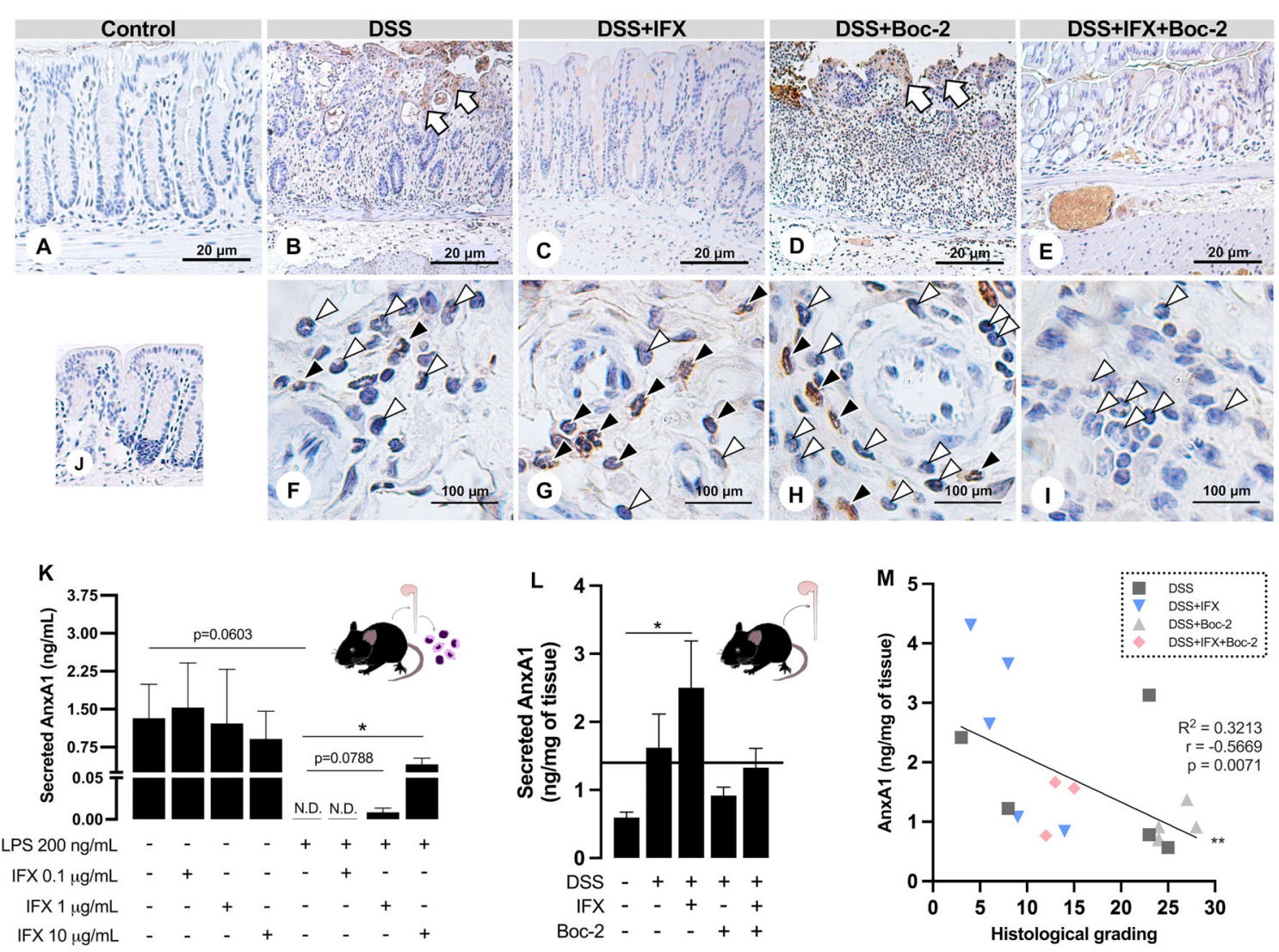

FIGURE 4 | Infliximab (IFX) induces annexin A1 (AnXA1) expression and secretion by infiltrated leukocytes during inflammation. (A-E) AnxA1 expression in the epithelial barrier from wild-type (WT) mice colon. AnxA1 concentrated on the wound bed (white arrows). (F-I) Immunostaining for AnxA1 in submucosal leukocytes. Tissue leukocytes positive (black arrowheads) or negative (white arrowheads) for AnxA1 staining. (J) Reaction control. Counterstaining, hematoxylin; embedding, paraffin; sections, $3 \mu \mathrm{m}$. Bars, $20 \mu \mathrm{m}$ (A-E) and $100 \mu \mathrm{m}$ (F-I). (K) AnxA1 secreted by lamina propria leukocytes cultured ex vivo. (L) AnxA1 secreted by colon explants in culture. (M) Moderate negative correlation between AnxA1 secreted into the tissue and histological grading. N.D., non-detected. ${ }^{\star} p<0.05$, ${ }^{* \star} p<0.01$. $n=3-4$ mice/group (K); $n=4-6$ mice/group (L). Results are expressed as the mean \pm SEM.

In the control epithelium, AnxA1 was weakly detected (Figure 4A). Colitis upregulated AnxA1 in the damaged epithelium from the DSS and DSS+Boc-2 groups (Figures $\mathbf{4 B}$, D). Mice treated with IFX, with or without FPR signaling, presented epithelial AnxA1 similar to the control group (Figures 4C, E). In turn, AnxA1 was weakly detected in tissue leukocytes in non-treated colitis (Figure 4F). After IFX, a high number of infiltrated AnxA1positive immune cells were observed (Figure 4G). AnxA1 was also detected in leukocytes under Boc-2 (Figure $\mathbf{4 H}$ ). Interestingly, in mice treated with IFX and Boc-2, most tissue leukocytes were negative for AnxA1 (Figure 4I).

Because the intracellular expression of AnxA1 was upregulated in tissue leukocytes upon IFX, we hypothesized that transmigrated immune cells could be important sources of AnxA1 for the inflamed tissue treated with this anti-TNF- $\alpha$. Using LPS in vitro assays, we simulated an inflammatory response in immune cells isolated from the colon of naive mice. When treated with increasing concentrations of IFX (0.1, 1.0 , and $10 \mu \mathrm{g} / \mathrm{ml}$ ), we detected a dose-dependent augmentation in AnxA1 secretion to the milieu (Figure 4K). This phenomenon was reproduced in the DSS-induced colitis treated with IFX, as colon explant supernatants had increased levels of AnxA1 compared to the control group (Figure 4L). Finally, we observed a moderate negative correlation between the secreted AnxA1 and the grading for histological damage of the colon (Figure 4M). These results suggest that IFX induces AnxA1 expression and secretion by tissue leukocytes, and this mechanism is linked to tissue repair.

\section{DISCUSSION}

Previously, we reported the relevance of AnxA1 in the efficacy of IFX during acute colitis in female Balb/c mice (8). Here, we corroborate it using mice of different strain and sex and focusing on the late phase of disease. On biopsies from CD patients, we further confirm that IFX modulates AnxAl in positive responders. Moreover, we describe FPR1 as a potential marker of tissue homeostasis after IFX treatment. Our results suggest that IFX stimulates activated intestinal leukocytes to express and 
secrete AnxA1 to the inflamed milieu, where it is free to bind to FPRs and promote tissue repair (29).

Although biologicals are the most successful drug class at inducing remission among the therapies classically used to treat IBD (30), they still burden patients with heterogeneous outcomes, including hypersensitivity, allergies, lack of responsiveness, or progressive loss of efficacy over the lifetime (31). Even clinically remittent patients may present remaining mucosal inflammation associated with severe mucosal disease activity (32). In response to this, the follow-up protocol for treated patients proposes the assessment of histological remission as a more reliable tool to predict a sustained and steroid-free clinical remission, with lower need for hospitalization and surgery (33). Herein, we observed a marked expression of AnxA1 in the colon of CD patients who underwent remission after IFX. For AnxA1 and FPR1, high tissue levels correlated with lower histological damage. In a general manner, the literature on IBD suggests that AnxA1 secretion is evoked during the active disease. Failures in this mechanism contribute to a more severe and prolonged disease $(26,34)$. Similarly, therapeutic interventions have a better outcome when capable of increasing AnxA1 levels $(8,14)$. In the inflamed site, AnxA1 binding to FPRs mediates the resolution of inflammation and mucosal homeostasis $(16,28)$. The data we present here support the mechanisms described above. Moreover, they suggest that tissue FPR1 and AnxA1 could constitute useful tools for following up the disease activity and the efficacy of IFX in CD patients.

Mucosal inflammation begins with the opening of the epithelial barrier after injury, which allows the translocation of microbial agents. Once the commensal microbiota is detected by the immune cells that patrol the tissue, an inflammatory response is assembled to contain the spreading of bacteria to the blood and other tissues. However, an unbalanced inflammation feed-forwards tissue damage and increases bacterial translocation (35). Upon injury or death, columnshaped intestinal epithelial cells can lose contact with each other, assuming a flat morphology. The organism responds by triggering a proliferation of basal crypt cells and epithelial migration, promoting wound closure (36). By binding FPRs, full-length AnxA1 and its peptide, Ac2-26, stimulate the migration of epithelial cells and wound healing $(15,16)$. Ac226 binding to FPR1 induces oxidative inactivation of phosphatase and tensin homolog protein and protein tyrosine phosphatase-PEST, phosphorylating focal adhesion kinase and paxillin, consequently stimulating cells to migrate (15). Additionally, endogenous AnxA1 acts as an anchor between cytoplasmic $\beta$-actin and the plasma membrane, whereas the AnxA1-FPR2 axis inhibits RhoA GTPase activity, stabilizing the cytoskeleton (37). Our results confirmed that the lack of endogenous AnxA1 is very detrimental to the progression of experimental colitis: AnxA1 $1^{-1-}$ mice had much worse clinical and histological outcomes, fewer intestinal regulatory $\mathrm{T}$ cell (Treg) counts, and a $25 \%$ mortality rate compared to WT mice. Preventing AnxA1 and other agonists from binding with FPRs in vivo was almost equally harmful as all clinical parameters were impaired and tissue damage was remarkable, especially considering crypt and epithelial loss. Interestingly, treatment with IFX did not compensate for the absence of endogenous AnxA1 or FPR signaling. The blockade of the AnxA1-FPR axis impaired crypt restitution, whereas a lack of AnxA1 impacted the ability of IFX to promote wound healing and to rebalance the immune response. Based on these data, we propose that protection of the epithelial barrier with IFX must be complemented by the downstream pathways resulting from the binding of AnxA1 (and other endogenous agonists) to FPRs (Figure 5).

It is well known that the use of IFX on chronic diseases modulates cellular pathways downstream of TNF receptors (TNFRs) by blocking soluble and transmembrane TNF- $\alpha$ (38, 39). Considering the complexity of the mechanisms evoked by IFX and the heterogeneous responses it produces, other endogenous players are likely to be involved in successful outcomes. Herein, we suggest that AnxA1 expression might be one mechanism induced by IFX, which may, in turn, regulate its effects at some level. This notion is supported by the modulation of intracellular and secreted AnxA1 after IFX treatment in colitic mice. In colonic mucosal and submucosal leukocytes activated by inflammatory mediators, IFX evoked AnxA1 expression. The same group had increased secretion of AnxA1, which was most likely being provided by those leukocytes. In line with this, increased levels of AnxA1 were secreted as higher doses of IFX were added to LPSstimulated intestinal leukocytes ex vivo. These results seem to be translatable to the human condition because we also observed increased AnxA1 in biopsies from CD patients in association with histological remission. Indeed, the lack of TNF- $\alpha$ signaling through TNFR1 in mice during colitis favors tissue AnxA1 expression and increases the frequency of $\mathrm{CD}^{+}$and $\mathrm{CD}^{+} \mathrm{T}$ lymphocytes positive for AnxA1 (26). These data indicate that intestinal immune cells are the major sources of secreted AnxA1; this could be one resolutive mechanism of IFX that was not previously described. Interestingly, the Boc-2 groups had less secreted AnxA1 with or without IFX. This suggests a more complex mechanism of action for IFX, in which proper FPR signaling is required to induce AnxA1 secretion by leukocytes and, in turn, to mediate tissue repair and wound healing.

Obviously, FPR agonists other than AnxA1 could be involved in the efficacy of IFX. In the inflammatory milieu, FPR ligands can be found among endogenous metabolites, peptides, and damage- and pathogen-associated molecular patterns (DAMPs and PAMPs, respectively) (40). These include $N$-formyl-peptides from mitochondrial damage (41), the antimicrobial peptide LL37 and lipoxin A4 (22). It should be noted that the bacterialderived peptide formyl-methyl-leucine-phenylalanine (fMLP) can be present at high concentrations in the wounded intestinal tissue (42) and binds FPR1 with a strong affinity, triggering healing pathways on epithelial cells $(20,43)$. Despite that, this work addresses AnxA1 participation because of its pivotal roles in IBD. Our choice was also supported by the increased AnxA1 secretion upon IFX in experimental colitis and its correlation with tissue recovery. It is also important to point out that, although we argue that AnxA1 and FPRs contribute to 


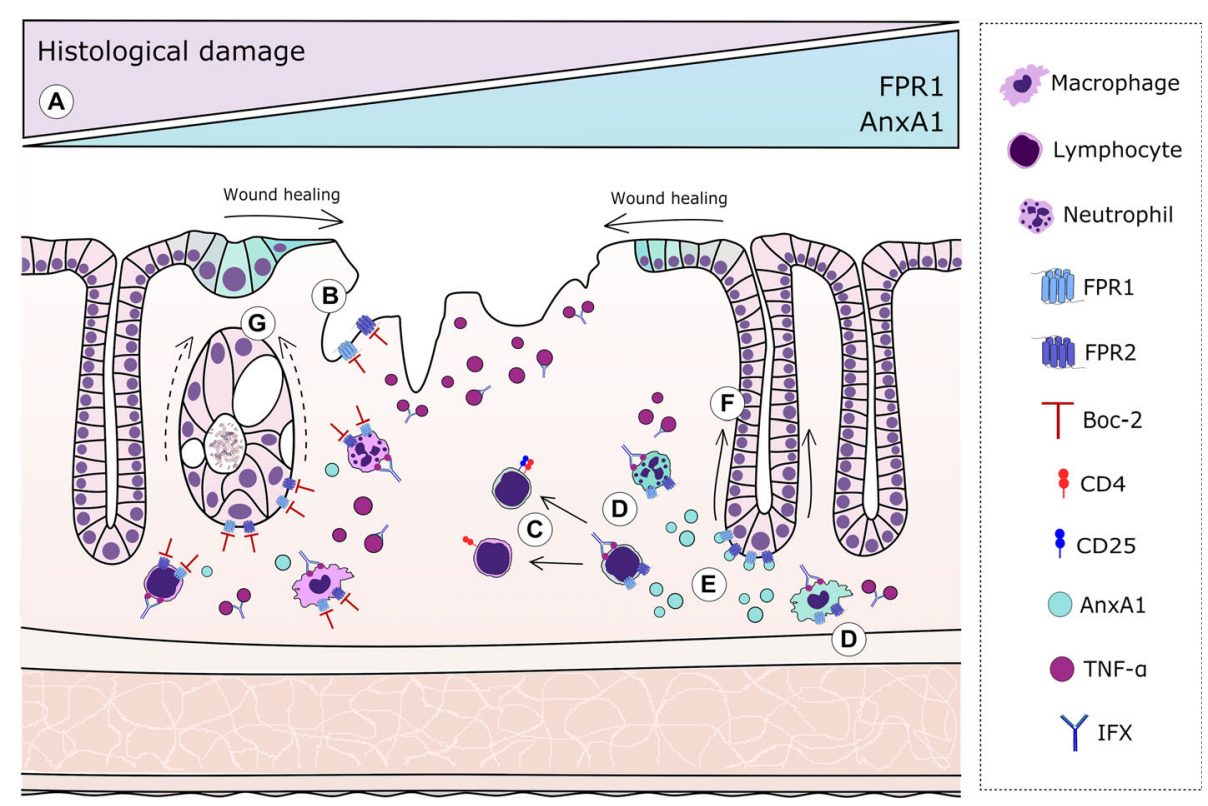

FIGURE 5 | Graphical representation of the resolutive effects of the annexin A1 (AnxA1)-formyl peptide receptor (FPR) axis complementary to infliximab (IFX) mechanisms. In humans: (A) Increased expressions of FPR1 and AnxA1 in colonic epithelial cells and lamina propria leukocytes correlate with histological remission after IFX. In mice: (B) AnxA1 expression is evoked in epithelial cells from damaged areas, but blockage of FPRs impairs proper re-epithelization. (C) In tissue T lymphocytes, the absence of endogenous AnxA1 skews cells to a more pro-inflammatory profile, resulting in the decreased number of regulatory $T$ cells (Tregs). (D) Immune cells transmigrated to the intestinal tissue are important sources of AnXA1 after IFX. (E) IFX induces AnxA1 secretion to the inflammatory milieu, which correlates with histological recovery. (F) In IFX-treated colitis, proper signaling of FPRs is crucial to adequate crypt regeneration. (G) Blockade of FPRs impairs the ability of IFX to protect glandular cells and restore the characteristic $U$-shape of crypts, producing amorphous structures after loss of basal-apical orientation and $\beta$ actin/villin distribution.

the efficacy of IFX, it is possible that they might be involved in the effects of other therapies as well. We do not claim that this modulation is exclusive to IFX, but that it obtains for this specific therapy. We hope that our study encourages others to explore therapies that might be complemented by AnxA1-FPR, elucidating possible mechanisms of therapeutic failure in IBD. We believe that the data we presented here may contribute to the identification of poor responsiveness cases, leading to long-term remission based on the pivotal homeostatic roles of AnxA1 through FPRs.

\section{DATA AVAILABILITY STATEMENT}

The original contributions presented in the study are included in the article/Supplementary Material. Further inquiries can be directed to the corresponding author.

\section{ETHICS STATEMENT}

The studies involving human participants were reviewed and approved by the boards on ethics from Santa Casa and University of São Paulo. The patients/participants provided their written informed consent to participate in this study. The animal study was reviewed and approved by the Committee on Ethics of Animal Experiments, University of São Paulo.

\section{AUTHOR CONTRIBUTIONS}

MS and SF conceived the hypothesis, designed the study, interpreted the data, and prepared the manuscript. MS, GR, $\mathrm{MB}, \mathrm{SS}$, and RL designed and carried out the experiments. MS, $\mathrm{MQ}$, and AV participated in patient approach and collecting samples and medical history. SO, MP, and SF provided scientific supervision, infrastructure, and essential tools to perform experiments. All authors contributed to the article and approved the submitted version.

\section{FUNDING}

This work was supported by Fundação de Amparo a Pesquisa do Estado de São Paulo, Brazil (grant nos. FAPESP 2014/07328-4, 2016/19682-2 and 2019/02806-9).

\section{ACKNOWLEDGMENTS}

The authors thank Dextran Products Limited (Canada) and Janssen-Cilag Pharmaceutics (Argentina) for kindly 
providing DSS and Remicade ${ }^{\circledR}$, respectively. We thank the medical and nursing staff from Santa Casa School of Medical Sciences (Brazil) for collecting the blood samples. Confocal analyses were supported by CMR Advanced Bio-Imaging Facility (UK).

\section{REFERENCES}

1. Abraham C, Medzhitov R. Interactions Between the Host Innate Immune System and Microbes in Inflammatory Bowel Disease. Gastroenterology (2011) 140:1729-37. doi: 10.1053/j.gastro.2011.02.012

2. Klotz U, Teml A, Schwab M. Clinical Pharmacokinetics and Use of Infliximab. Clin Pharmacokinet (2007) 46:645-60. doi: 10.2165/00003088200746080-00002

3. Berns M, Hommes DW. Anti-TNF- $\alpha$ Therapies for the Treatment of Crohn's Disease: The Past, Present and Future. Expert Opin Investig Drugs (2016) 25:129-43. doi: 10.1517/13543784.2016.1126247

4. Costa J, Magro F, Caldeira D, Alarcão J, Sousa R, Vaz-Carneiro A. Infliximab Reduces Hospitalizations and Surgery Interventions in Patients With Inflammatory Bowel Disease: A Systematic Review and Meta-Analysis. Inflamm Bowel Dis (2013) 19:2098-110. doi: 10.1097/MIB.0b013e31829936c2

5. Fratila OC, Craciun C. Ultrastructural Evidence of Mucosal Healing After Infliximab in Patients With Ulcerative Colitis. J Gastrointest Liver Dis (2010) 19:147-53.

6. Veyrard P, Boschetti G, Nancey S, Roblin X. Predictive Models of Therapeutic Response to Vedolizumab: A Novel Step Into Personalized Medicine in Crohn's Disease? Inflamm Bowel Dis (2018) 24:1193-5. doi: 10.1093/ibd/ izy033

7. Scaldaferri F, Pecere S. Emerging Mechanisms of Action and Loss of Response to Infliximab in Ibd: A Broader Picture. Biochem Pharmacol Open Access (2015) 05:1-9. doi: 10.4172/2167-0501.1000206

8. de Paula-Silva M, Barrios BE, Macció-Maretto L, Sena AA, Farsky SHP, Correa SG, et al. Role of the Protein Annexin A1 on the Efficacy of Anti-TNF Treatment in a Murine Model of Acute Colitis. Biochem Pharmacol (2016) 115:104-13. doi: 10.1016/j.bcp.2016.06.012

9. Ferraro B, Leoni G, Hinkel R, Ormanns S, Paulin N, Ortega-Gomez A, et al. Pro-Angiogenic Macrophage Phenotype to Promote Myocardial Repair. J Am Coll Cardiol (2019) 73:2990-3002. doi: 10.1016/j.jacc.2019.03.503

10. de Jong R, Leoni G, Drechsler M, Soehnlein O. The Advantageous Role of Annexin A1 in Cardiovascular Disease. Cell Adhes Migr (2017) 11:261-74. doi: 10.1080/19336918.2016.1259059

11. Colamatteo A, Maggioli E, Azevedo Loiola R, Hamid Sheikh M, Calì G, Bruzzese D, et al. Reduced Annexin A1 Expression Associates With Disease Severity and Inflammation in Multiple Sclerosis Patients. J Immunol (2019) 203:1753-65. doi: 10.4049/jimmunol.1801683

12. Headland SE, Jones HR, Norling LV, Kim A, Souza PR, Corsiero E, et al. Neutrophil-Derived Microvesicles Enter Cartilage and Protect the Joint in Inflammatory Arthritis. Sci Transl Med (2015) 7:1-25. doi: 10.1126/ scitranslmed.aac5608

13. Kamal AM, Flower RJ, Perretti M. An Overview of the Effects of Annexin 1 on Cells Involved in the Inflammatory Process. Mem Inst Oswaldo Cruz (2005) 100:39-48. doi: 10.1590/S0074-02762005000900008

14. Sena A, Grishina I, Thai A, Goulart L, Macal M, Fenton A, et al. Dysregulation of Anti-Inflammatory Annexin A1 Expression in Progressive Crohns Disease. PloS One (2013) 8:1-13. doi: 10.1371/journal.pone.0076969

15. Leoni G, Alam A, Alexander Neumann P, Lambeth JD, Cheng G, McCoy J, et al. Annexin A1, Formyl Peptide Receptor, and NOX1 Orchestrate Epithelial Repair. J Clin Invest (2013) 123:443-54. doi: 10.1172/JCI65831

16. Leoni G, Neumann PA, Kamaly N, Quiros M, Nishio H, Jones HR, et al. Annexin A1-Containing Extracellular Vesicles and Polymeric Nanoparticles Promote Epithelial Wound Repair. J Clin Invest (2015) 125:1215-27. doi: 10.1172/JCI76693.dination

17. Reischl S, Troger J, Jesinghaus M, Kasajima A, Wilhelm DF, Friess H, et al. Annexin A1 Expression Capacity as a Determinant for Disease Severity in Crohn's Disease. Dig Dis (2020) 38:398-407. doi: 10.1159/000505910

\section{SUPPLEMENTARY MATERIAL}

The Supplementary Material for this article can be found online at: https://www.frontiersin.org/articles/10.3389/fimmu.2021. 714138/full\#supplementary-material

18. Perretti M, D'Acquisto F. Annexin A1 and Glucocorticoids as Effectors of the Resolution of Inflammation. Nat Rev Immunol (2009) 9:62-70. doi: 10.1038/ nri2470

19. Tsai YF, Yang SC, Hwang TL. Formyl Peptide Receptor Modulators: A Patent Review and Potential Applications for Inflammatory Diseases (2012-2015). Expert Opin Ther Pat (2016) 26:1139-56. doi: 10.1080/13543776.2016.1216546

20. Babbin BA, Jesaitis AJ, Ivanov AI, Kelly D, Laukoetter M, Nava P, et al. Formyl Peptide Receptor-1 Activation Enhances Intestinal Epithelial Cell Restitution Through Phosphatidylinositol 3-Kinase-Dependent Activation of Rac1 and Cdc42. J Immunol (2007) 179:8112-21. doi: 10.4049/jimmunol.179.12.8112

21. Wentworth CC, Jones RM, Kwon YM, Nusrat A, Neish AS. CommensalEpithelial Signaling Mediated via Formyl Peptide Receptors. Am J Pathol (2010) 177:2782-90. doi: 10.2353/ajpath.2010.100529

22. Vong L, Ferraz JGPP, Dufton N, Panaccione R, Beck PL, Sherman PM, et al. Up-Regulation of Annexin-A1 and Lipoxin A4 in Individuals With Ulcerative Colitis may Promote Mucosal Homeostasis. PloS One (2012) 7:1-9. doi: 10.1371/journal.pone.0039244

23. Birkl D, O’Leary MN, Quiros M, Azcutia V, Schaller M, Reed M, et al. Formyl Peptide Receptor 2 Regulates Monocyte Recruitment to Promote Intestinal Mucosal Wound Repair. FASEB J (2019) 33:13632-43. doi: 10.1096/ fj.201901163R

24. Best WR, Becktel JM, Singleton JW, Kern F, Kern F. Development of a Crohn's Disease Activity Index: National Cooperative Crohn's Disease Study. Gastroenterology (1976) 70:439-44. doi: 10.1016/S0016-5085(76)80163-1

25. Laharie D, Reffet A, Belleannée G, Chabrun E, Subtil C, Razaire S, et al. Mucosal Healing With Methotrexate in Crohns Disease: A Prospective Comparative Study With Azathioprine and Infliximab. Aliment Pharmacol Ther (2011) 33:714-21. doi: 10.1111/j.1365-2036.2010.04569.x

26. Sena AA, Pedrotti LP, Barrios BE, Cejas H, Balderramo D, Diller A, et al. Lack of TNFRI Signaling Enhances Annexin A1 Biological Activity in Intestinal Inflammation. Biochem Pharmacol (2015) 98:1-10. doi: 10.1016/j.bcp.2015.09.009

27. Alam A, Leoni G, Wentworth CC, Kwal JM, Wu H, Ardita CS, et al. Redox Signaling Regulates Commensal-Mediated Mucosal Homeostasis and Restitution and Requires Formyl Peptide Receptor. Mucosal Immunol (2014) 7:645-55. doi: 10.1038/mi.2013.84

28. Babbin B, Laukoetter M, Nava P, Koch S, Lee W, Capaldo C, et al. Annexin A1 Regulates Intestinal Mucosal Injury, Inflammation, and Repair. J Immunol (2008) 181:5035-44. doi: 10.4049/jimmunol.181.7.5035

29. Khau T, Langenbach SY, Schuliga M, Harris T, Johnstone CN, Anderson RL, et al. Annexin-1 Signals Mitogen-Stimulated Breast Tumor Cell Proliferation by Activation of the Formyl Peptide Receptors (FPRs) 1 and 2. FASEB J (2011) 25:483-96. doi: 10.1096/fj.09-154096

30. Magro F, Portela F. Management of Inflammatory Bowel Disease With Infliximab and Other Anti-Tumor Necrosis Factor Alpha Therapies. BioDrugs (2010) 24:3-14. doi: 10.2165/11586290-000000000-00000

31. Pichler WJ. Adverse Side-Effects to Biological Agents. Allergy Eur J Allergy Clin Immunol (2006) 61:912-20. doi: 10.1111/j.1398-9995.2006.01058.x

32. Baars JE, Nuij VJAA, Oldenburg B, Kuipers EJ, van der Woude CJ. Majority of Patients With Inflammatory Bowel Disease in Clinical Remission Have Mucosal Inflammation. Inflamm Bowel Dis (2012) 18:1634-40. doi: 10.1002/ibd.21925

33. Pineton de Chambrun G, Blanc P, Peyrin-Biroulet L. Current Evidence Supporting Mucosal Healing and Deep Remission as Important Treatment Goals for Inflammatory Bowel Disease. Expert Rev Gastroenterol Hepatol (2016) 10:915-27. doi: 10.1586/17474124.2016.1174064

34. Leoni G, Nusrat A. Annexin A1: Shifting the Balance Towards Resolution and Repair. Biol Chem (2016) 397:971-9. doi: 10.1515/hsz-2016-0180

35. Rogler G. Resolution of Inflammation in Inflammatory Bowel Disease. Lancet Gastroenterol Hepatol (2017) 2:521-30. doi: 10.1016/S2468-1253(17)30031-6 
36. Iizuka M, Konno S. Wound Healing of Intestinal Epithelial Cells. World J Gastroenterol (2011) 17:2161-71. doi: 10.3748/wjg.v17.i17.2161

37. Cristante E, McArthur S, Mauro C, Maggioli E, Romero IA, Wylezinska-Arridge $\mathrm{M}$, et al. Identification of an Essential Endogenous Regulator of Blood-Brain Barrier Integrity, and Its Pathological and Therapeutic Implications. Proc Natl Acad Sci USA (2013) 110:832-41. doi: 10.1073/pnas.1209362110

38. Ten Hove T, Van Montfrans C, Peppelenbosch MP, Van Deventer SJH. Infliximab Treatment Induces Apoptosis of Lamina Propria T Lymphocytes in Crohn's Disease. Gut (2002) 50:206-11. doi: 10.1136/gut.50.2.206

39. Pedersen J, Coskun M, Soendergaard C, Salem M, Nielsen OH. Inflammatory Pathways of Importance for Management of Inflammatory Bowel Disease. World J Gastroenterol (2014) 20:64-77. doi: 10.3748/wjg.v20.i1.64

40. Migeotte I, Communi D, Parmentier M. Formyl Peptide Receptors: A Promiscuous Subfamily of G Protein-Coupled Receptors Controlling Immune Responses. Cytokine Growth Factor Rev (2006) 17:501-19. doi: 10.1016/j.cytogfr.2006.09.009

41. He HQ, Ye RD. The Formyl Peptide Receptors: Diversity of Ligands and Mechanism for Recognition. Molecules (2017) 22:1-33. doi: 10.3390/ molecules22030455

42. Leoni G, Gripentrog J, Lord C, Riesselman M, Sumagin R, Parkos CA, et al. Human Neutrophil Formyl Peptide Receptor Phosphorylation and the Mucosal Inflammatory Response. J Leukoc Biol (2015) 97:87-101. doi: 10.1189/jlb.4A0314-153R
43. Wentworth CC, Alam A, Jones RM, Nusrat A, Neish AS. Enteric Commensal Bacteria Induce Extracellular Signal-Regulated Kinase Pathway Signaling via Formyl Peptide Receptor-Dependent Redox Modulation of Dual Specific Phosphatase. J Biol Chem (2011) 286:3844855. doi: 10.1074/jbc.M111.268938

Conflict of Interest: The authors declare that the research was conducted in the absence of any commercial or financial relationships that could be construed as a potential conflict of interest.

Publisher's Note: All claims expressed in this article are solely those of the authors and do not necessarily represent those of their affiliated organizations, or those of the publisher, the editors and the reviewers. Any product that may be evaluated in this article, or claim that may be made by its manufacturer, is not guaranteed or endorsed by the publisher.

Copyright (C) 2021 de Paula-Silva, da Rocha, Broering, Queiroz, Sandri, Loiola, Oliani, Vieira, Perretti and Farsky. This is an open-access article distributed under the terms of the Creative Commons Attribution License (CC BY). The use, distribution or reproduction in other forums is permitted, provided the original author(s) and the copyright owner(s) are credited and that the original publication in this journal is cited, in accordance with accepted academic practice. No use, distribution or reproduction is permitted which does not comply with these terms. 